Article

\title{
Distribution of $N$-Methylimidazole in Ionic Liquids/Organic Solvents Systems
}

\author{
Milen G. Bogdanov * and Ivan Svinyarov \\ Faculty of Chemistry and Pharmacy, Sofia University St.Kl. Ohridski, 1 J. Bourchierblvd., 1164 Sofia, Bulgaria; \\ ivansvinyarov@abv.bg \\ * Correspondence: mbogdanov@chem.uni-sofia.bg; Tel.: +359-2-81-61-228; Fax: +359-2-96-25-438
}

Received: 26 August 2017; Accepted: 20 September 2017; Published: 22 September 2017

\begin{abstract}
The partition coefficients, $K_{\operatorname{mim}}$, of $N$-methylimidazole (mim) in two-component systems composed of ionic liquid (IL) and a series of organic solvents immiscible with ILs (butyl acetate, ethyl acetate, tert-butyl methyl ether, diethyl ether and cyclohexane) were determined by a shake-flask method. The influence of different factors such as temperature, solvent polarity, mim concentration, and water content on $K_{\text {mim }}$ by using 1-butyl-3-methylimidazolium chloride $\left\{\left[C_{4} C_{1} i m\right] C l\right\}$ as a model compound was comprehensively studied. The calculated thermodynamic functions of transfer $\left(\Delta_{\operatorname{tr}} G^{0}\right.$, $\Delta_{\mathrm{tr}} H^{0}, \Delta_{\mathrm{tr}} S^{0}$ ) showed that the mim migration (IL $\rightarrow$ organic phase) is a thermodynamically unfavorable and enthalpy-determined process in the temperature range of 298.15 to $328.15 \mathrm{~K}$; however, the results suggested that mim partition toward the organic phase can be enhanced by the rational manipulation of the extraction conditions. Experiments conducted with other 1-alkyl-3-methylimidazolim chlorides $\left(\mathrm{C}_{\mathrm{n}} \mathrm{C}_{1} \mathrm{im}\right] \mathrm{Cl}(\mathrm{n}=6,8,10)$ revealed that mim possesses similar behavior and can be successfully washed out from the ILs by extraction with organic solvents. The results obtained in this study give some clues toward the choice of an appropriate solvent and conditions to be employed for the purification of halide-based ILs by means of a liquid-liquid extraction.
\end{abstract}

Keywords: ionic liquids; liquid-liquid extraction; separation; $\mathrm{N}$-methylimidazole; partition coefficient; interactions; transfer

\section{Introduction}

The interest in ionic liquids (ILs) has been continuously growing in the last two decades due to their unique properties and high potential as "green" alternatives to conventional harmful organic solvents [1-6]. Among others, a very special property of these "designers' solvents" is that they can be tailored to fulfill certain requirements by simple variation of the ions. In order to address this issue, many researchers focused their efforts on understanding the behavior of ILs at a molecular level and on establishing structure-properties relationships that allow for the rational design of ILs for specific applications [7-12]. It is noteworthy that the ILs' properties are strongly dependent on their purity [12-14]; therefore, the proper purification of ILs is a factor of immense importance, which allows consistent results to be obtained by different research groups. It is well established that the purification of ILs is more difficult than that for common volatile organic solvents, and that the success in this process is dependent on the attention devoted to each aspect of their production, including the starting materials' purity, reaction conditions, post-treatment of the reaction mixtures, etc. The approach most applied for the synthesis of ILs is based on the quaternization reaction between appropriate $N_{-}, S_{-}$, or $P$-containing compounds and haloalkanes (mainly chlorides and bromides) followed by metathesis reaction in order for the halide anion to be further exchanged [15]. In this way, the production of halide-based ILs of high purity is a precondition for purer ILs to be obtained at the end of the reaction scheme. The quaternization step can be carried out both under solvent-free conditions or in the presence of appropriate solvents [15-20]. Usually, this first step is conducted at temperatures up to 
$80{ }^{\circ} \mathrm{C}$, since reverse or side reactions that contribute negatively to the quality of the target IL take place at higher temperatures [18]. Some authors also suggest the above reactions to be conducted at a lower temperature in order to avoid the formation of colored impurities [20]. Unfortunately, this approach results in a limited extent of transformation and prolonged reaction times due to the constant increase of the reaction medium viscosity upon IL formation and reduced reactivity at low temperatures. Regardless of the chosen way of synthesis, the resulted halide-based ILs have to be properly purified. The main "contaminants" at that stage are the corresponding residual organic base and haloalkane [21,22], and their removal is typically accomplished by multiple liquid-liquid extractions with an organic solvent, followed by prolonged heating of the product under reduced pressure.It is noteworthy that, depending on the boiling points of the residuals and their affinity toward the IL, the entire procedure might require a repetitive performance, thus prolonging the time and increasing the consumption of organic solvents, particularly when one works on a multi-gram scale. Considering the short chain haloalkanes, they are highly volatile and it is unlikely that they will remain in the final IL sample after heating under reduced pressure. In contrast, alkylamines used for the synthesis, particularly $N$-methylimidazole, tend to have high boiling points and thus are not as easily removed in vacuo; hence, these are better removed by repetitive washing of the IL with organic solvents.

The partition coefficient of a species between two immiscible liquid phases can be considered as an important physicochemical parameter from an engineering perspective, since it can be useful in the recovery of compounds by liquid-liquid extraction [23-31]. In our present study [32], we introduced an improved apparatus for continuous liquid-liquid extraction to be used as a tool for the purification of halide-based ionic liquids after the quaternization step. The apparatus employs the heat of the evaporating solvent to heat up the extraction chamber, thus allowing for the process to be conducted at elevated temperatures. The purification carried out with this tool proved successful in the removal of impurities such as residual starting compounds under mild conditions. It was also shown to give ILs of high purity in a shorter time and with significantly reduced consumption of organic solvent as compared with the common procedure [32]. By using this liquid-liquid extraction approach, the following purification step consisting of prolonged heating under deep vacuum can be considerably shortened, because of the lower boiling point and the affinity of the nonpolar organic solvents to the IL [33] in comparison to that of the $N$-methylimidazole or some of the commonly used haloalkanes in the quaternization reaction. In continuation of this study, herein we present a more in-depth investigation on the distribution of $\mathrm{N}$-methylimidazole ( $\mathrm{mim}$ ) in biphasic systems composed of ILs and IL-immiscible organic solvents. The influence of different factors such as temperature, solvent type and polarity, mim concentration, and water content on the partition coefficients, $K_{\mathrm{mim}}$, by using 1-butyl-3-methylimidazolium chloride $\left\{\left[\mathrm{C}_{4} \mathrm{C}_{1} \mathrm{im}\right] \mathrm{Cl}\right\}$ as a model compound was comprehensively studied. Thermodynamic functions of transfer (standard Gibbs energy $\left(\Delta_{\operatorname{tr}} G^{0}\right)$, standard enthalpy $\left(\Delta_{\operatorname{tr}} H^{0}\right)$, and standard entropy $\left.\left(\Delta_{\operatorname{tr}} S^{0}\right)\right)$ were calculated and used for a rational discussion of the mim migration from the IL phase to the organic phase. Additional preliminary data for other 1-alkyl-3-methylimidazolim chlorides $\left(\mathrm{C}_{n} \mathrm{C}_{1} \mathrm{im}\right] \mathrm{Cl}(\mathrm{n}=6,8,10)$ were also reported. The results obtained in this study give some hints toward the choice of an appropriate solvent and conditions to be employed for the purification of halide-based ILs by means of a liquid-liquid extraction.

\section{Materials and Methods}

All organic chemicals used in this study were purchased from Sigma-Aldrich (FOT, Bulgaria) and were of analytical grade. Selected properties of materials, including solvents and ILs, are listed in Table 1 . The ILs studied were 1-alkyl-3-methylimidazolium chlorides, $\left[C_{n} C_{1} i m\right] C l(n=4,6,8,10)$, and were synthesized, purified, and characterized by the authors as described elsewhere [18]. Their structure and purity were unequivocally proven by ${ }^{1} \mathrm{H}-$ and ${ }^{13} \mathrm{C}-\mathrm{NMR}$ spectroscopy [34]. All ILs were dried under reduced pressure and stored under inert atmosphere. $N$-Methylimidazole content was analyzed by means of HPLC. 
Table 1. Polarity $\left(E_{T} N\right)$, dipolarity $(S d P)$, acidity $(S A)$, and basicity $(S B)$ of solvents used in this study ${ }^{1}$.

\begin{tabular}{ccccc}
\hline Materials (Abbreviation) & $E_{T} N$ & $S d P$ & $S A$ & $S B$ \\
\hline Water & 1.000 & 0.997 & 1.062 & 0.025 \\
N-methylimidazole (mim) & n.a. $^{2}$ & 0.959 & 0.069 & 0.658 \\
butyl acetate (BuOAc) & 0.241 & 0.535 & 0 & 0.525 \\
ethyl acetate (EtOAc) & 0.228 & 0.603 & 0 & 0.542 \\
tert-butyl methyl & 0.124 & 0.422 & 0 & 0.567 \\
ether (MTBE) & 0.117 & 0.385 & 0 & 0.562 \\
diethyl ether $\left(\mathrm{Et}{ }_{2} \mathrm{O}\right)$ & 0.006 & 0 & 0 & 0.073 \\
Cyclohexane & 0.614 & 1.172 & 0.167 & 0.869 \\
{$\left[\mathrm{C}_{4} \mathrm{C}_{1}\right.$ im]Cl } & 0.562 & 1.139 & 0.159 & 0.932 \\
{$\left[\mathrm{C}_{6} \mathrm{C}_{1}\right.$ im]Cl } & 0.549 & 1.096 & 0.159 & 1.001 \\
{$\left[\mathrm{C}_{8} \mathrm{C}_{1}\right.$ im]Cl } & n.a. & 1.115 & 0.159 & 0.932 \\
{$\left[\mathrm{C}_{10} \mathrm{C}_{1}\right.$ im $] \mathrm{Cl}$} &
\end{tabular}

${ }^{1}$ Data was extracted from References [35-40]; ${ }^{2}$ n.a. refers to not available.

The experiments for measuring of partition coefficients of $\mathrm{N}$-methylimidazole in the IL/solvent systems were performed in graduated microtubes $(2 \mathrm{~mL})$. The dried ILs were contaminated with a known amount of mim and were put in contact with the corresponding solvents in a volume ratio of 1:1. The shaking time was set to $5 \mathrm{~min}$, since it was found that prolonged shaking (up to $2 \mathrm{~h}$ ) gave similar equilibrium results. The mixtures were allowed to separate for $3 \mathrm{~h}$ at the temperature studied, then centrifuged for better phase separation, and aliquots from the two phases were subjected to analysis. All experiments were performed in duplicate, with a variation in results of less than $5 \%$. The corresponding partition coefficients, $K_{\mathrm{mim}}$, and thermodynamic parameters of the transfer $\mathrm{IL} \rightarrow$ organic phase $\left(\Delta_{\mathrm{tr}} G^{0}, \Delta_{\mathrm{tr}} H^{0}, \Delta_{\mathrm{tr}} S^{0}\right)$ were calculated according to Equations (1)-(4):

$$
\begin{gathered}
K_{\mathrm{mim}}=C_{\mathrm{org}} / C_{\mathrm{IL}} \\
\Delta_{\mathrm{tr}} G^{0}=-R T \ln \left(K_{\mathrm{mim}}\right) \\
\Delta_{\mathrm{tr}} G^{0}=\Delta_{\mathrm{tr}} H^{0}-T \Delta_{\mathrm{tr}} S^{0} \\
\ln \left(K_{\mathrm{mim}}\right)=-\Delta_{\mathrm{tr}} H^{0} / R \times 1 / T+\Delta_{\mathrm{tr}} S^{0} / R
\end{gathered}
$$

where $C_{\text {org }}$ and $C_{\mathrm{IL}}$ are the concentrations of mim in the organic phase and the IL phase, respectively, $R$ is the universal gas constant $\left(8.314 \mathrm{~J} \mathrm{~mol}^{-1} \mathrm{~K}^{-1}\right)$, and $T$ is the temperature $(\mathrm{K})$.

\section{Results}

At the very beginning of this work, we studied the temperature dependence of mim partitioning in IL/organic solvent systems within the temperature range of 298.15-328.15 K (see Figure 1a). The solvents used for this experiments were butyl acetate, ethyl acetate, tert-butyl methyl ether, and cyclohexane, and $\left[\mathrm{C}_{4} \mathrm{C}_{1} \mathrm{im}\right] \mathrm{Cl}$ was the model IL. On the basis of the $K_{\operatorname{mim}}$ obtained and by using

\begin{tabular}{|c|c|c|c|c|c|c|c|c|}
\hline TemperatureK & $K_{\operatorname{mim}}$ & $\ln \left(K_{\operatorname{mim}}\right)$ & $\begin{array}{c}\Delta_{\mathrm{tr}} G^{0} \mathrm{~kJ} \\
\mathrm{~mol}^{-1}\end{array}$ & $\begin{array}{c}\Delta_{\mathrm{tr}} H^{0} \mathrm{~kJ} \\
\mathrm{~mol}^{-1}\end{array}$ & $\begin{array}{l}\Delta_{\mathrm{tr}} S^{0} \mathrm{~J} \\
\mathrm{~mol}^{-1}\end{array}$ & $\begin{array}{c}T \Delta_{\mathrm{tr}} S^{0} \mathrm{~kJ} \\
\mathrm{~mol}^{-1}\end{array}$ & $\zeta_{\mathrm{Htr}} 1 \%$ & $\zeta_{\text {TStr }} 2 \%$ \\
\hline \multicolumn{9}{|c|}{ Butyl Acetate } \\
\hline 298.15 & 0.860 & -0.15 & 0.38 & 1.52 & 3.83 & 1.14 & 57 & 43 \\
\hline 308.15 & 0.900 & -0.11 & 0.34 & & & & & \\
\hline 318.15 & 0.840 & -0.17 & 0.30 & & & & & \\
\hline 328.15 & 0.940 & -0.06 & 0.26 & & & & & \\
\hline
\end{tabular}
Equations (2)-(4), the thermodynamic parameters of transfer (standard Gibbs energy $\left(\Delta_{\operatorname{tr}} G^{0}\right)$, standard enthalpy $\left(\Delta_{\mathrm{tr}} H^{0}\right)$, and standard entropy $\left.\left(\Delta_{\mathrm{tr}} S^{0}\right)\right)$ were calculated and are summarized in Table 2.

Table 2. Partition coefficients and thermodynamics functions of mim transfer IL $\rightarrow$ organic solvent. 
Table 2. Cont.

\begin{tabular}{|c|c|c|c|c|c|c|c|c|}
\hline TemperatureK & $K_{\text {mim }}$ & $\ln \left(K_{\operatorname{mim}}\right)$ & $\begin{array}{c}\Delta_{\mathrm{tr}} G^{0} \mathrm{~kJ} \\
\mathrm{~mol}^{-1}\end{array}$ & $\begin{array}{c}\Delta_{\mathrm{tr}} H^{0} \mathrm{~kJ} \\
\mathrm{~mol}^{-1}\end{array}$ & $\begin{array}{l}\Delta_{\mathrm{tr}} S^{0} \mathrm{~J} \\
\mathrm{~mol}^{-1}\end{array}$ & $\begin{array}{c}T \Delta_{\mathrm{tr}} S^{0} \mathrm{~kJ} \\
\mathrm{~mol}^{-1}\end{array}$ & $\zeta_{\mathrm{Htr}}{ }^{1 \%}$ & $\zeta_{\text {TStr }}{ }^{2 \%}$ \\
\hline \multicolumn{9}{|c|}{ Ethyl Acetate } \\
\hline 298.15 & 0.586 & -0.53 & 1.32 & 10.18 & 29.69 & 8.85 & 53 & 47 \\
\hline 308.15 & 0.662 & -0.41 & 1.03 & & & & & \\
\hline 318.15 & 0.780 & -0.25 & 0.73 & & & & & \\
\hline 328.15 & 0.841 & -0.17 & 0.43 & & & & & \\
\hline \multicolumn{9}{|c|}{ Correlationequation: $\ln \left(K_{\operatorname{mim}}\right)=3.75-1224 \times 1 / T ; R^{2}=0.99$} \\
\hline \multicolumn{9}{|c|}{ tert-Butyl Methyl Ether } \\
\hline 298.15 & 0.117 & -2.15 & 5.38 & 17.18 & 39.59 & 11.80 & 59 & 41 \\
\hline 308.15 & 0.138 & -1.98 & 4.98 & & & & & \\
\hline 318.15 & 0.177 & -1.73 & 4.59 & & & & & \\
\hline 328.15 & 0.217 & -1.53 & 4.19 & & & & & \\
\hline \multicolumn{9}{|c|}{ Correlationequation: $\ln \left(K_{\operatorname{mim}}\right)=4.76-2067 \times 1 / T ; R^{2}=0.99$} \\
\hline \multicolumn{9}{|c|}{ Cyclohexane } \\
\hline 298.15 & 0.018 & -4.02 & 9.97 & 27.39 & 58.41 & 17.42 & 61 & 39 \\
\hline 308.15 & 0.025 & -3.71 & 9.39 & & & & & \\
\hline 318.15 & 0.038 & -3.26 & 8.81 & & & & & \\
\hline 328.15 & 0.048 & -3.05 & 8.22 & & & & & \\
\hline \multicolumn{9}{|c|}{ Correlationequation: $\ln \left(K_{\operatorname{mim}}\right)=7.02-3295 \times 1 / T ; R^{2}=0.99$} \\
\hline
\end{tabular}

Parameters $\zeta_{\mathrm{Htr}}$ and $\zeta_{\mathrm{Str}}$ were used to describe the relative fraction of enthalpy and entropy of transfer: ${ }^{1} \% \zeta_{\mathrm{Htr}}=$ $\left(\Delta_{\mathrm{tr}} H^{0} /\left(\Delta_{\mathrm{tr}} H^{0}+\mathrm{T} \cdot \Delta_{\mathrm{tr}} S^{0}\right)\right) \cdot 100 ;{ }^{2} \% \zeta_{\mathrm{Str}}=\left(\Delta_{\mathrm{tr}} S^{0} /\left(\Delta_{\mathrm{tr}} H^{0}+\mathrm{T} \Delta_{\mathrm{tr}} S^{0}\right)\right) \cdot 100$.

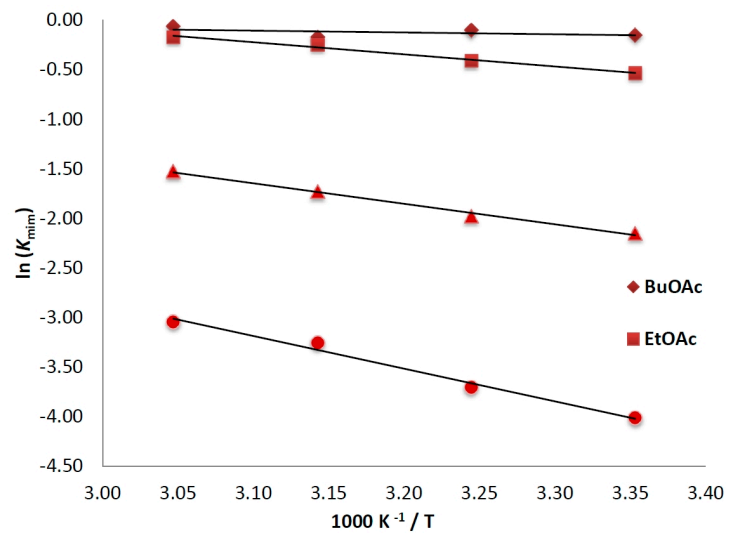

(a)

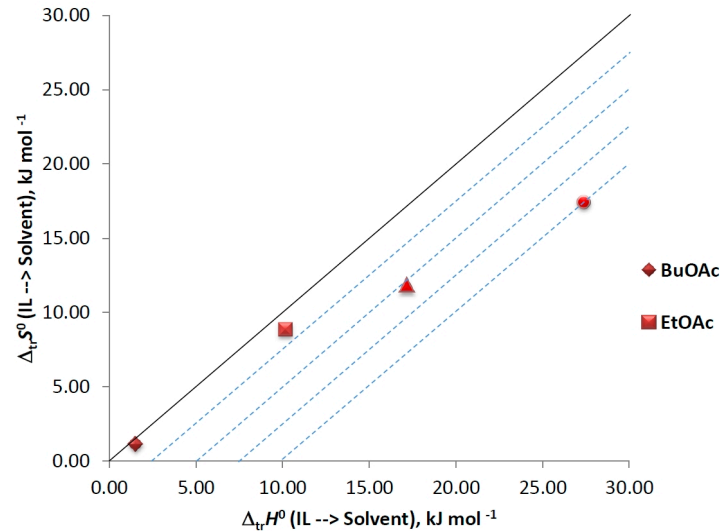

(b)

Figure 1. (a) van't Hoff plots of $\ln \left(K_{\mathrm{mim}}\right)$ vs. $1 / \mathrm{T}$ for mim in a systems $\left[\mathrm{C}_{4} \mathrm{C}_{1} \mathrm{im}\right] \mathrm{Cl} /$ organic solvent; (b) Relationship between the enthalpic and entropic terms of transfer functions from $\left[\mathrm{C}_{4} \mathrm{C}_{1} \mathrm{im}\right] \mathrm{Cl}$ to solvent for $N$-methylimidazole at $298.15 \mathrm{~K}$. The isoenergetic curves of $\Delta_{\mathrm{tr}} G^{0}$ function are marked by dotted lines.

A short overview of the results presented in Table 2 reveals a number of notable regularities. First, the calculated $K_{\mathrm{mim}}<1$ for all systems show the preferential partition of mim in the IL-phase. It was also found that $K_{\text {mim }}$ measured at same temperature, e.g., $298 \mathrm{~K}$, strongly depends on the organic solvent used, following the order BuOAc (0.860) > EtOAc (0.586) > MTBE (0.117) > cyclohexane (0.018), and that this order stays unchanged regardless of the temperature applied. Regarding the temperature effect, $K_{\text {mim }}$ were found to increase for all systems with the increase of temperature. It is noteworthy that this phenomenon is most pronounced for the less polar cyclohexane $\left(K_{\operatorname{mim}}\right.$ enhancement of $\left.167 \%\right)$ and less pronounced for the solvent of the highest polarity-BuOAc $\left(K_{\operatorname{mim}}\right.$ enhancement of $\left.12 \%\right)$. The van't Hoff plots $\ln \left(K_{\mathrm{mim}}\right)$ vs. $1 / \mathrm{T}$ exhibit linearity for all systems (see Figure $\left.1 \mathrm{a}\right)$, which indicates that the standard enthalpy of transfer of mim is temperature-independent [23] in the temperature range studied. The calculated $\Delta_{\operatorname{tr}} G^{0}$ and $\Delta_{\operatorname{tr}} H^{0}$ values are positive for all systems; consequently, the partition 
of mim from the IL-phase to the organic phase is a thermodynamically unfavorable and endothermic structure-breaking process. The positive $\Delta_{\mathrm{tr}} H^{0}$ is also an indication for stronger interactions between mim and the IL ions, the latter being realized by hydrogen bonding (see Section 4). In addition, $\Delta_{\mathrm{tr}} H^{0}$ was found to increase with the decrease of the solvent polarity, following the order: BuOAc $(1.52)<\operatorname{EtOAc}(10.18)<\operatorname{MTBE}(17.18)<$ cyclohexane (27.39). The latter suggests that the energy required for the mim migration toward the organic phase can be minimized by employing a solvent of higher polarity. The transfer entropy $\Delta_{\mathrm{tr}} S^{0}$ is also positive in all cases. This indicates the higher mobility of the species in the transfer process, as well as the positive influence of that term on $\Delta_{\operatorname{tr}} G^{0}$. Similar to the enthalpy, $\Delta_{\mathrm{tr}} S^{0}$ has a reverse relationship with the solvent polarity, as indicated by the following order: BuOAc (3.83) < EtOAc (29.69) < MTBE (39.59) < cyclohexane (58.41), and this can be attributed to the decreased number of interactions in the solvents of lower polarity. The contribution of different factors to the partition of mim can be assessed by means of a graphical approach [41]. The region of the diagram presented as Figure $1 \mathrm{~b}$, where $\Delta_{\mathrm{tr}} H^{0}>0 ; \mathrm{T} \Delta_{\mathrm{tr}} S^{0}>0 ; \Delta_{\mathrm{tr}} H^{0}>\mathrm{T} \Delta_{\mathrm{tr}} S^{0}$, i.e., sector VII, corresponds to the enthalpy-determined processes, and sector VIII, where $\Delta_{\mathrm{tr}} H^{0}>0 ; \mathrm{T} \Delta_{\mathrm{tr}} S^{0}>0 ; \Delta_{\mathrm{tr}} H^{0}<\mathrm{T} \Delta_{\mathrm{tr}} S^{0}$, corresponds to the entropy-determined process. Isoenergetic curves of the $\Delta_{\mathrm{tr}} G^{0}$ function are marked as dotted lines in this figure. As can be seen, all data points are situated in the same sector, sector VII, which means that the transfer processes of mim for the organic solvents obey same mechanism, which is enthalpy-determined. The minimum energy difference for the process is observed for $\operatorname{BuOAc}\left(\Delta_{\mathrm{tr}} G^{0}=0.38 \mathrm{~kJ} \mathrm{~mol}^{-1}\right)$, and the maximum for cyclohexane $\left(\Delta_{\mathrm{tr}} G^{0} 9.97 \mathrm{~kJ} \mathrm{~mol}^{-1}\right)$, while $\Delta_{\mathrm{tr}} G^{0}$ is additionally lowered by the increase of the temperature. To sum up, the removal of residual $\mathrm{N}$-methylimidazole from $\left[\mathrm{C}_{4} \mathrm{C}_{1} \mathrm{im}\right] \mathrm{Cl}$ by means of a liquid-liquid extraction is an entropy-driven, structure-breaking, not spontaneous process, which can be facilitated by employing solvents of high polarity and applying elevated temperatures.

The concentration of a solute recovered from a solvent by means of a liquid-liquid extraction decreases in a continuous manner after each extractive cycle. In order to investigate the influence of this factor on the partition of mim, we contaminated IL samples with different amounts of mim and performed extractions with organic solvents. Because these experiments were conducted at ambient conditions, diethyl ether $\left(\mathrm{Et}_{2} \mathrm{O}\right)$ was also included as an IL-immiscible solvent. The results obtained are presented in Figure 2a. As it can be seen, the extraction ability of the solvents is consistent with the results presented above, i.e., the mim partition into the organic phase is facilitated by an organic solvent with higher polarity according to the following order: BuOAc $<\mathrm{EtOAc}<\mathrm{MTBE} \approx \mathrm{Et}_{2} \mathrm{O}<$ cyclohexane (for the sake of clarity, the latter was omitted from Figure $2 \mathrm{a}, K_{\operatorname{mim}}=0.018$ ). Further, the solvents can be divided on two categories. The first one consists of esters-BuOAc and EtOAc, for which $K_{\text {mim }}$ increases with the increase of mim concentration, and the second one-ethers and cyclohexane, for which $K_{\mathrm{mim}}$ is concentration-independent. A rough extrapolation of these results suggests that the polar solvents are more efficient at higher mim concentrations, whereas all organic solvents can be equally used for the removal of mim in low concentrations.

Another factor that might change the ILs' properties is the presence of water [13,14]. To study the influence of the water content in ILs, we measured the partition of mim by using BuOAc, EtOAc, and MTBE as organic solvents. As can be seen from Figure $2 b, K_{\operatorname{mim}}$ decreases with the increase of water concentration for the three solvents used, and this phenomenon has a reverse relationship to the extraction ability of the latter. The observed effect is most pronounced for the BuOAc ( $80 \%$ reduction), best performed under "dry conditions", and less pronounced for MTBE (45\% reduction) upon increasing the water content from 0 to $20 \%(w t / w t)$. It is also noteworthy that the presence of even $5 \%$ of water changes the order of the solvents' ability to extract mim; consequently, the water content can be considered as a factor of an immense importance that controls the mim partition in a significant manner, and that the use of a dry solvent is preferred in the purification of ILs. 


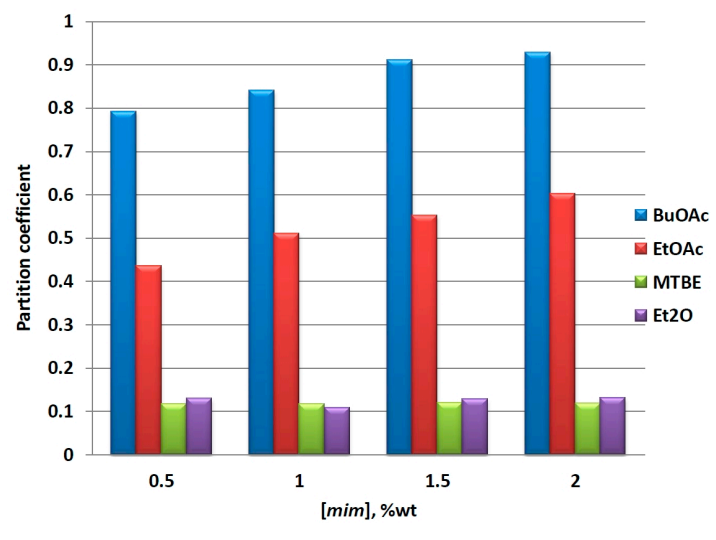

(a)

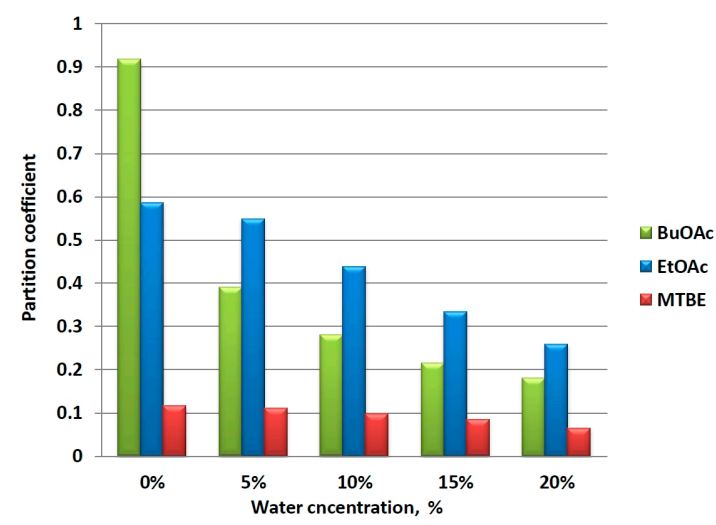

(b)

Figure 2. (a) Effect of mim concentration and (b) water content on the partition of mim in a $\left[\mathrm{C}_{4} \mathrm{C}_{1} \mathrm{im}\right] \mathrm{Cl}$ /organic solventsystem.

\section{Discussion}

The results presented above showed that the transfer of mim from the IL to the organic phase is not a spontaneous process, and that is dependent on factors such as solvent type and polarity, temperature, mim concentration, and water content. In order for these results to be rationalized, a short description of the ILs' structure both in the pure state and in the presence of other molecules should be given.

Pure ILs can be considered as self-assembly amphiphiles, which form H-bonded-polymeric networks as a general structural pattern for both solid and liquid phases [42,43]. The introduction of other species into pure ILs, however, disrupts this H-bond network [44] and generates different secondary nano-structures with polar and non-polar domains, which in turn affects ILs' properties such as polarity, viscosity, reactivity, and solvating ability, to name just a few $[2,12-14,18,37]$. In case of dilution with solvents, depending on the solvent polarity and H-bonding ability, supramolecular aggregates, triple ions, contact ion pairs, or solvent-separated ions can be formed [42]. Regarding 1-alkyl-3-methyimidazolium-based ILs, there are three main binding sites in the imidazolium ion capable of participating as hydrogen bond donors, and these are at positions $\mathrm{H}-\left({ }^{4} \mathrm{C}\right), \mathrm{H}-\left({ }^{5} \mathrm{C}\right)$, and $\mathrm{H}-\left({ }^{2} \mathrm{C}\right)$. Indeed, it was shown that the IL anion prefers to bind to the proton at the $\left({ }^{2} \mathrm{C}\right)$ position, the latter being the most acidic amongst the others [45-47], and that the introduction of a solute/ solvent to the IL leads to a competition for the active sites of both ions, the outcome of which strongly depends on the differences between the acidic/basic properties of the participants [44-52]. In other words, solutes with pronounced acidic properties will compete with the imidazolium ion to form H-bonds with $\mathrm{Cl}^{-}$, whereas those with basic properties will compete with the chloride anion for the acidic sites of the cation. A short overview of the data presented in Table 1 shows that the organic solvents used in this study have zero acidity and that their basicity is almost equal to each other (an exception is cyclohexane); consequently, the second scenario will take place when an IL interacts with them. Considering the lower basicity of the solvents in comparison to that of the ILs, and the fact that this property of ILs is dominated by the anion [37], it can be assumed that $\mathrm{Cl}^{-}$will win the competition for the position at $\mathrm{H}-\left({ }^{2} \mathrm{C}\right)$, thus pushing solvent molecules to interact with $\mathrm{H}-\left({ }^{4} \mathrm{C}\right)$ and $\mathrm{H}-\left({ }^{5} \mathrm{C}\right)$. In this way, the presence of hydrogen-bonded species "solvent-IL" in the IL-phase can be suggested. In contrast to that, the existence of such complexes in the organic phase is not expected due to the negligible solubility of ILs in these solvents [51].

Having this simplified description of the systems under study, our next goal is to explain the results for the mim partition. It is evident from Table 2 that $K_{\text {mim }}$ is always lower than unity, which suggests that "mim-IL" interactions are rather preferable to "mim-solvent" and "solvent-IL" interactions. This suggestion can be supported by the fact that mim possesses pronounced basicity and weak acidity. By a comparison of the solvent basicity (SB) and solvent acidity (SA) values of all 
participants in the competition (see Table 1), it can be concluded that mim will compete both with $\mathrm{Cl}^{-}$and solvent molecules for the acidic binding sites of the imidazolium ion. Simultaneously, mim will offer alternative $\mathrm{H}$-binding centers to the $\mathrm{Cl}^{-}$, which will additionally strengthen the mim-IL interactions and will hinder its transfer to the organic phase. Based on the above reasoning, it can be concluded that the organic solvent, which can extract mim from the IL phase in a more efficient manner, is able to substitute it from the H-bond embedded mim-IL network. Considering the basic properties of the compounds under study, however, it can be seen that this is not the case here, and that another approach based on different types of interactions that facilitate the formation of "mim-solvent" complexes has to be given in order for the apparent partition coefficients to be explained. It was observed that $K_{\mathrm{mim}}$ strongly depends on the solvent polarity, decreasing in the following order: $\mathrm{BuOAc}>\mathrm{EtOAc}>\mathrm{MTBE} \approx \mathrm{Et}_{2} \mathrm{O}>$ cyclohexane, regardless of the temperature applied. Interestingly, we obtained a very good linear correlation between the averaged $K_{\operatorname{mim}}$ values and the solvents' polarities expressed in the Reichardt's $E_{T} N$ scale $\left(\left(\ln \left(K_{\operatorname{mim}}\right)=a \times E_{T} N+b, R^{2}=0.994\right)\right.$. On the one hand, this relationship gives some clues toward the mechanism of extraction, and on the other, it can be used as a tool for solvent selection in the liquid-liquid extraction of mim, i.e., IL-immiscible solvents, possessing higher $E_{T} N$ value are expected to be more efficient in this process. In 2009, Catalan showed [38] that the Reichardt polarity parameter [40] is composed of several components, including solvent dipolarity $(S d P)$, acidity $(S A)$, and basicity $(S B)$, and that its value is mainly determined by the $S A$ term, followed by $S d P$ and $S B$, with contributing factors of 2:1:0.3, respectively. Based on the above reasoning, and taking into account the zero acidity and equal basicity of the solvents used in this study, we can conclude that the solvent dipolarity can be regarded as the main factor that governs the mim transfer from the IL phase to the organic solvent. However, as it can be seen from Table 1, BuOAc has a lower $S d P$ value than that of EtOAc, but performs the best in the extraction of mim in the absence of water. This can be attributed to the increased hydrophobicity of the former due to the presence of two additional methylene groups in its structure. Consequently, the solvent hydrophobicity, which seems to be reflected by the $E_{T} N$ polarity parameter, also plays an important role in the extraction of mim. In support of the conclusions made above is the data obtained for the thermodynamic parameters of transfer (see Table 2), which reveals that the enthalpy $\Delta_{\mathrm{tr}} H^{0}$ increases with the decrease of the solvent polarity, following the order:BuOAc $(1.52)<$ EtOAc $(10.18)<$ MTBE $(17.18)<$ cyclohexane (27.39). Furthermore, despite the fact that it is entropy favored $\left(\Delta_{\mathrm{tr}} S^{0}>0\right)$, it was found that the process of mim transfer toward the organic phase is not spontaneous $\left(\Delta_{\mathrm{tr}} G^{0}>0\right)$, but is endothermic $\left(\Delta_{\mathrm{tr}} H^{0}>0\right)$ and enthalpy-determined $\left(\Delta_{\mathrm{tr}} H^{0}>\mathrm{T} \Delta_{\mathrm{tr}} S^{0}\right)$ for all systems studied (see Figure $\left.1 \mathrm{~b}\right)$. This data can also be employed to clarify what the driving force of the process is by analysis of the signs of the thermodynamic parameters. Usually, $\Delta H>0$ and $\Delta S>0$ imply a hydrophobic association; $\Delta H<0$ and $\Delta S<0$ reflect van der Waals forces or hydrogen bond formation; and $\Delta H<0$ and $\Delta S>0$ suggest an electrostatic force. Considering this data, it can be seen that the transfer of mim is entropy-driven with a main driving force of hydrophobic interactions between the organic solvent and mim, which is in line with the above discussion about the H-bonding ability of mim and its influence on the partition. Moreover, if one calculates the thermodynamic parameters of mim transfer for the reverse process, i.e., solvent $\rightarrow \mathrm{IL}$, one can see that both $\Delta_{\mathrm{tr}} H^{0}$ and $\Delta_{\mathrm{tr}} S^{0}$ are negative, which suggests a process that is governed by the formation of hydrogen bonds.

The above discussion is also consistent with the ILs' behavior at different temperatures. It was put forward by Skarmoutsos et al. [53] that the H-bonds network in pure ILs is continuously reorganizing, and that the number and lifetime of the H-bonds between the ions of ILs, particularly for $\left[\mathrm{C}_{4} \mathrm{C}_{1} \mathrm{im}\right] \mathrm{Cl}$, are strongly dependent on the temperature. The authors showed that in a hot IL, H-bonds are mainly formed between two ions and are rapidly breaking and reforming, whereas in a cold IL, a higher proportion of bifurcated state $\mathrm{H}$-bonds occurs, with a dramatic extension of the lifetime of individual $\mathrm{H}$-bonds [53]. In this way, the observed increase of $K_{\mathrm{mim}}$ with the increase of temperature seen in this study can be attributed to the disruption of the H-bond-embedded network at high temperatures, which allows the hydrophobic interactions between mim and the solvent to take place in a more 
efficient manner, hence facilitating the transfer of the former to the organic phase. In contrast to that, the addition of a polar species with pronounced acidic properties, e.g., water (see Table 1), will increase the number of centers capable of participating as H-bond donors in the IL-phase. It was shown [46,47] that the water molecules preferably bind to the $\mathrm{Cl}^{-}$anion, which lowers it basicity and allows mim to form stronger $\mathrm{H}$-bonds with the IL cation by changing its position from the less acidic $\mathrm{H}-\left({ }^{4} \mathrm{C}\right)$ and $\mathrm{H}-\left({ }^{5} \mathrm{C}\right)$ to the more acidic $\mathrm{H}-\left({ }^{2} \mathrm{C}\right)$ at low water concentrations, and to interact with water itself at higher concentrations. This stronger structure reduces the ability of the solvent molecules to efficiently solvate mim, thus lowering the respective $K_{\text {mim }}$ (Figure $2 b$ ).

Changing the alkyl chain length in the ILs ions can be considered a direct way to tune their hydrophobic properties [54,55]. 1-Alkyl-3-methylimidazolium chlorides with alkyl chain lengths varying from 4 to 10 were chosen to conduct a preliminary study on the influence of that factor on the mim partition in IL/organic solvents biphasic systems. The corresponding experimental data is presented in Figure 3. It is noteworthy that the solubility of the organic solvents in ILs increases with the increase of the alkyl chain length, which causes deviation in the phase ratio solvent to IL, e.g., from 1:1 in the case of $\left[\mathrm{C}_{4} \mathrm{C}_{1} \mathrm{im}\right] \mathrm{Cl}$ to 0.4:1.6 in the case of $\left[\mathrm{C}_{10} \mathrm{C}_{1} \mathrm{im}\right] \mathrm{Cl}$ when BuOAc or EtOAc were used as organic solvents. Furthermore, MTBE was completely soluble in $\left[\mathrm{C}_{10} \mathrm{C}_{1} \mathrm{im}\right] \mathrm{Cl}$ at a mixing ratio of 1:1. Therefore, for comparison purposes, the results are presented and discussed in terms of extraction efficiency $(E E \%)$, the latter being defined as: $E E \%=\left[K_{\mathrm{mim}} /\left(1+1 / R_{V}\right)\right] \times 100$, thus taking into account the apparent phase ratio $R_{V}$.

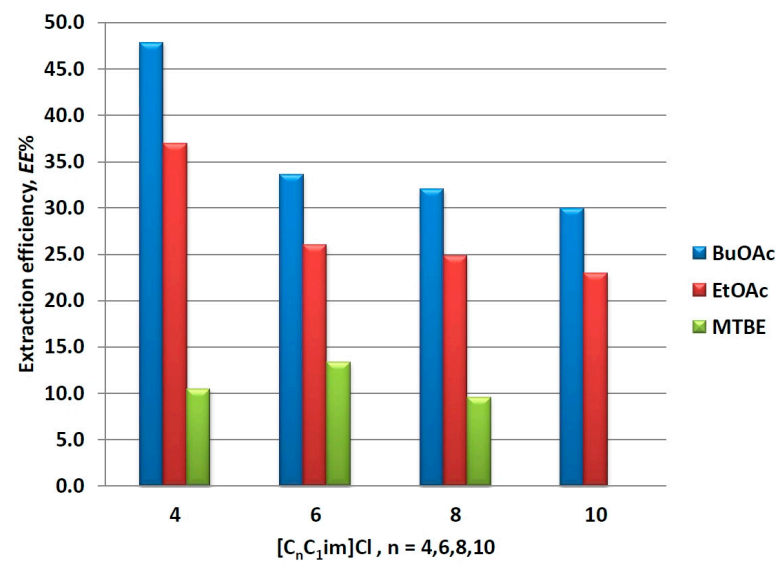

Figure 3. Effect of alkyl side chain in the imidazolium ion on mim partition in different IL/solvent systems.

As it can be seen from Figure 3, the above observed order of extraction ability of the organic solvents stays intact $(\mathrm{BuOAc}>\mathrm{EtOAc}>\mathrm{MTBE})$ regardless of the IL. Further, $E E \%$ decreases upon increasing the number of carbon atoms in the alkyl side chain, and this change is again more pronounced for the better performed solvents (BuOAc and EtOAc). The latter phenomenon can be attributed to the increasing hydrophobicity of ILs upon the inclusion of additional methylene groups [54,55], which enhance the hydrophobic interactions in the IL phase and hinder the unopposed transfer of mim for the organic phase. On the other hand, the increased solubility of the solvent into ILs also can contribute to the apparent reduction of extraction ability. Nevertheless, the results presented here suggest that mim can be successfully washed out from the ILs by extraction with organic solvents such as BuOAc, EtOAc, and MTBE.

\section{Conclusions}

This article represents a comprehensive study on the influence of different factors such as solvent type and polarity, temperature, solute concentration, and water content on the partition 
of $N$-methylimidazole in biphasic systems of ILs and organic solvents (butyl acetate, ethyl acetate, tert-butyl methyl ether, diethyl ether, and cyclohexane). By using the thermodynamic functions of transfer $\left(\Delta_{\mathrm{tr}} G^{0}, \Delta_{\mathrm{tr}} H^{0,} \Delta_{\mathrm{tr}} S^{0}\right)$, we showed that the mim transfer (IL $\rightarrow$ organic phase) is a thermodynamically unfavorable, structure-breaking process, and that mim is predominantly partitioned into the IL regardless of the conditions applied. However, mim transfer toward the organic phase can be facilitated by employing solvents of higher polarity and by the elevation of the temperature. The presence of water in the system has a negative effect on the process of transfer, the latter suggesting that the use of dry solvents is preferred in the purification of ILs. The results obtained in the present study contribute to the understanding of ILs' behavior at a molecular level and give some clues toward the rational selection of solvents and conditions to be employed for the purification of halide-based ILs by means of a liquid-liquid extraction, which is a precondition for purer ILs to be obtained after metathesis reaction. This can be of great importance from an applied standpoint, since a considerable amount of time and energy could be economized.

Acknowledgments: The financial support of the National Science Fund of Bulgaria at the Ministry of Education and Science (project DFNI T 02/23) is greatly acknowledged. The authors also would like to acknowledge the contribution of the COST Action CM1206-Exchange on Ionic Liquids.

Author Contributions: I.S. and M.B. contributed equally to this work and have approved the final manuscript.

Conflicts of Interest: The authors declare no conflict of interest.

\section{References}

1. Plechkova, N.V.; Seddon, K.R. Applications of ionic liquids in the chemical industry. Chem. Soc. Rev. 2008, 37, 123-150. [CrossRef] [PubMed]

2. Hallett, J.P.; Welton, T. Room-temperature ionic liquids: Solvents for synthesis and catalysis. 2. Chem. Rev. 2011, 111, 3508-3576. [CrossRef] [PubMed]

3. Smiglak, M.; Pringle, J.M.; Lu, X.; Han, L.; Zhang, S.; Gao, H.; MacFarlane, D.R.; Rogers, R.D. Ionic liquids for energy, materials, and medicine. Chem. Commun. 2014, 50, 9228-9250. [CrossRef] [PubMed]

4. Bogdanov, M.G. Ionic Liquids as Alternative Solvents for Extraction of Natural Products. In Alternative Solvents for Natural Products Extraction, Green Chemistry and Sustainable Technology; Chemat, F., VianAbert, M., Eds.; Springer: Berlin/Heidelberg, Germany, 2014; pp. 127-166.

5. Hijo, A.A.C.T.; Maximo, G.J.; Costa, M.C.; Batista, E.A.C.; Meirelles, A.J.A. Applications of ionic liquids in the food and bioproducts industries. ACS Sustain. Chem. Eng. 2016, 4, 5347-5369. [CrossRef]

6. Ventura, S.P.M.; eSilva, F.A.; Quental, M.V.; Mondal, D.; Freire, M.G.; Coutinho, J.A.P. Ionic-liquid-mediated extraction and separation processes for bioactive compounds: Past, present, and future trends. Chem. Rev. 2017, 117, 6984-7052. [CrossRef] [PubMed]

7. Raj, J.J;; Wilfred, C.D.; Shah, S.N.; Pranesh, M.; Mutalib, M.A.; Lethesh, K.C. Physicochemical and thermodynamic properties of imidazolium ionic liquids with nitrile and ether dual functional groups. J. Mol. Liq. 2017, 225, 281-289. [CrossRef]

8. Zhao, N.; Jacquemin, J. The development of the UNIFAC-CONDUCT model as a novel approach for the estimation of the conductivity of pure ionic liquids. Fluid PhaseEquilibr. 2017, 449, 60-67. [CrossRef]

9. Zhao, N.; Menegolla, H.B.; Degirmenci, V.; Wagner, Z.; Bendová, M.; Jacquemin, J. Group contribution method for evaluation of volumetric properties of ionic liquids using experimental data recommended by mathematical gnostics. Ind. Eng. Chem. Res. 2017, 56, 6827-6840. [CrossRef]

10. Zhao, Y.; Huang, Y.; Zhang, X.; Zhang, S. Quantitative prediction of viscosity of ionic liquids using $\mathrm{S}_{\sigma-\text { profile }}$ molecular descriptors. Phys. Chem. Chem. Phys. 2015, 17, 3761-3767. [CrossRef] [PubMed]

11. Coutinho, J.A.P.; Carvalho, P.J.; Oliveira, N.M.C. Predictive methods for the estimation of thermophysical properties of ionic liquids. RSC Adv. 2012, 2, 7322-7346. [CrossRef]

12. Bogdanov, M.G.; Kantlehner, W. Simple prediction of some physical properties of ionic liquids: The residual volume approach. Z. Naturforsch. B J. Chem. Sci. 2009, 64, 215-222. [CrossRef]

13. Widegren, J.A.; Laesecke, A.; Magee, J.W. The effect of dissolved water on the viscosities of hydrophobic room-temperature ionic liquids. Chem. Commun. 2005, 1610-1612. [CrossRef] [PubMed] 
14. Seddon, K.R.; Stark, A.; Torres, M.-J. Influence of chloride, water, and organic solvents on the physical properties of ionic liquids. Pure Appl. Chem. 2000, 72, 2275-2287. [CrossRef]

15. McIntosh, A.J.S.; Griffith, J.; Gräsvik, J. Methods of synthesis and purification of ionic liquids. In Application, Purification, and Recovery of Ionic Liquids; Kuzmina, O., Hallett, J.P., Eds.; Elsevier: Amsterdam, The Netherlands, 2016; pp. 59-99. Available online: https://doi.org/10.1016/B978-0-444-63713-0.00002-X (accessed on 21 September 2017).

16. Böwing, A.G.; Jess, A. Kinetics of single- and two-phase synthesis of the ionic liquid 1-butyl-3-methylimidazolium chloride. Green Chem. 2005, 7, 230-235. [CrossRef]

17. Chiappe, C.; Mezzetta, A.; Pomelli, C.S.; Puccini, M.; Seggiani, M. Product as reaction solvent: An unconventional approach for ionic liquid synthesis. Org. Process Res. Dev. 2016, 20, 2080-2084. [CrossRef]

18. Bogdanov, M.G.; Petkova, D.; Hristeva, S.; Svinyarov, I.; Kantlehner, W. New guanidinium-based room-temperature ionic liquids. Substituent and anion effect on density and solubility in water. Z. Naturforsch. B J. Chem. Sci. 2010, 65, 37-48. [CrossRef]

19. Clough, C.; Griffith, J.; Sulaiman, M.R.; Corbett, P.; Welton, T. Alkylation of 1-methylimidazole with 1-chlorobutane; the ionic liquid 1-butyl-3-methylimidazolium chloride. SCHEMSPIDER. [CrossRef]

20. Nockemann, P.; Binnemans, K.; Driesen, K. Purification of imidazolium ionic liquids for spectroscopic applications. Chem. Phys. Lett. 2005, 415, 131-136. [CrossRef]

21. Holbrey, J.D.; Seddon, K.S.; Wareing, R. A simple colorimetric method for the quality control of 1-alkyl-3-methylimidazolium ionic liquid precursors. Green Chem. 2001, 3, 33-36. [CrossRef]

22. Stark, A.; Behrend, P.; Braun, O.; Müller, A.; Ranke, J.; Ondruschka, B.; Jastorff, B. Purity specification methods for ionic liquids. Green Chem. 2008, 10, 1152-1161. [CrossRef]

23. Cláudio, A.F.M.; Freire, M.G.; Freire, C.S.R.; Silvestre, A.J.D.; Coutinho, J.A.P. Extraction of vanillin using ionic-liquid-based aqueous two-phase systems. Sep. Purif. Technol. 2010, 75, 39-47. [CrossRef]

24. Freire, M.G.; Teles, A.R.R.; Canongia Lopes, J.N.; Rebelo, L.P.N.; Marrucho, I.M.; Coutinho, J.A.P. Partition coefficients of alkaloids in biphasic ionic-liquid-aqueous systems and their dependence on the Hofmeister series. Sep. Sci. Technol. 2012, 47, 284-291. [CrossRef]

25. Tonova, K.; Svinyarov, I.; Bogdanov, M.G. Hydrophobic 3-alkyl-1-methylimidazolium saccharinates as extractants for L-lactic acid recovery. Sep. Purif. Technol. 2014, 125, 239-246. [CrossRef]

26. Cláudio, A.F.M.; Marques, C.F.C.; Boal-Palheiros, I.; Freire, M.G.; Coutinho, J.A.P. Development of back-extraction and recyclability routes for ionic-liquid-based aqueous two-phase systems. Green Chem. 2014, 16, 259-268. [CrossRef]

27. Bogdanov, M.G.; Keremedchieva, R.; Svinyarov, I. Ionic liquid-supported solid-liquid extraction of bioactive alkaloids. III. Ionic liquid regeneration and glaucine recovery from ionic liquid-aqueous crude extract of Glauciumflavum Cr. (Papaveraceae). Sep. Purif. Technol. 2015, 155, 13-19. [CrossRef]

28. Keremedchieva, R.; Svinyarov, I.; Bogdanov, M.G. Ionic liquid-based aqueous biphasic systems-A facile approach for ionic liquid regeneration from crude plant extracts. Processes 2015, 3, 769-778. [CrossRef]

29. Reyhanitash, E.; Zaalberg, B.; Kersten, S.R.A.; Schuur, B. Extraction of volatile fatty acids from fermented wastewater. Sep. Purif. Technol. 2016, 161, 61-68. [CrossRef]

30. Lima, Á.S.; Soares, C.M.F.; Paltram, R.; Halbwirth, H.; Bica, K. Extraction and consecutive purification of anthocyanins from grape pomace using ionic liquid solutions. Fluid PhaseEquilibr. 2017. [CrossRef]

31. Dimitrijević, A.; Ignjatović, L.; Tot, A.; Vraneš, M.; Zec, N.; Gadžurić, S.; Trtić-Petrović, T. Simultaneous extraction of pesticides of different polarity applying aqueous biphasic systems based on ionic liquids. J. Mol. Liq. 2017, 243, 646-653. [CrossRef]

32. Bogdanov, M.G.; Svinyarov, I. Efficient purification of halide-based ionic liquids by means of improved apparatus for continuous liquid-liquid extraction. Sep. Purif. Technol. 2017. [CrossRef]

33. Ren, S.; Hou, Y.; Wu, W.; Liu, W. Purification of ionic liquids: Sweeping solvents by nitrogen. J. Chem. Eng. Data 2010, 55, 5074-5077. [CrossRef]

34. Bogdanov, M.G.; Svinyarov, I.; Keremedchieva, R.; Sidjimov, A. Ionic liquid-supported solid-liquid extraction of bioactive alkaloids. I. New HPLC method for quantitative determination of glaucine in Glauciumflavum Cr. (Papaveraceae). Sep. Purif. Technol. 2012, 97, 221-227. [CrossRef]

35. Schade, A.; Behme, N.; Spange, S. Dipolarity versus polarizability and acidity versus basicity of ionic liquids as a function of their molecular structures. Chem. Eur. J. 2014, 20, 2232-2243. [CrossRef] [PubMed] 
36. Jessop, P.G.; Jessop, D.A.; Fu, D.; Phan, L. Solvatochromic parameters for solvents of interest in green chemistry. Green Chem. 2012, 14, 1245-1259. [CrossRef]

37. Ab Rani, M.A.; Brant, A.; Crowhurst, L.; Dolan, A.; Lui, M.; Hassan, N.H.; Hallett, J.P.; Hunt, P.A.; Niedermeyer, H.; Perez-Arlandis, J.M.; et al. Understanding the polarity of ionic liquids. Phys. Chem. Chem. Phys. 2011, 13, 16831-16840. [CrossRef] [PubMed]

38. Catalán, J. Toward a generalized treatment of the solvent effect based on four empirical scales: Dipolarity (SdP, a new scale), polarizability (SP), acidity (SA), and basicity (SB) of the medium. J. Phys. Chem. B 2009, 113, 5951-5960. [CrossRef] [PubMed]

39. Reichardt, C. Polarity of ionic liquids determined empirically by means of solvatochromicpyridinium $N$-phenolatebetaine dyes. Green Chem. 2005, 7, 339-351. [CrossRef]

40. Reichardt, C. Solvatochromic dyes as solvent polarity indicators. Chem. Rev. 1994, 94, 2319-2358. [CrossRef]

41. Perlovich, G.L. Thermodynamic approaches to the challenges of solubility in drug discovery and development. Mol. Pharm. 2014, 11, 1-11. [CrossRef] [PubMed]

42. Dupont, J. On the solid, liquid and solution structural organization of imidazolium ionic liquids. J. Braz. Chem. Soc. 2004, 15, 341-350. [CrossRef]

43. Greaves, T.L.; Drummond, C.J. Solvent nanostructure, the solvophobic effect and amphiphile self-assembly in ionic liquids. Chem. Soc. Rev. 2013, 42, 1096-1120. [CrossRef] [PubMed]

44. Cammarata, L.; Kazarian, S.G.; Salter, P.A.; Welton, T. Molecular states of water in room temperature ionic liquids. Phys. Chem. Chem. Phys. 2001, 3, 5192-5200. [CrossRef]

45. Ficke, L.E.; Brennecke, J.F. Interactions of ionic liquids and water. J. Phys. Chem. B 2010, 114, $10496-10501$. [CrossRef] [PubMed]

46. Khan, I.; Kurnia, K.A.; Mutelet, F.; Pinho, S.P.; Coutinho, J.A.P. Probing the interactions between ionic liquids and water: Experimental and quantum chemical approach. J. Phys. Chem. B 2014, 118, 1848-1860. [CrossRef] [PubMed]

47. Khan, I.; Taha, M.; Ribeiro-Claro, P.; Pinho, S.P.; Coutinho, J.A.P. Effect of the cation on the interactions between alkyl methylimidazolium chloride ionic liquids and water. J. Phys. Chem. B 2014, 118, 10503-10514. [CrossRef] [PubMed]

48. Zhang, Q.-G.; Wang, N.-N.; Wang, S.-L.; Yu, Z.-W. Hydrogen bonding behaviors of binary systems containing the ionic liquid 1-butyl-3-methylimidazolium trifluoroacetate and water/methanol. J. Phys. Chem. B 2011, 115, 11127-11136. [CrossRef] [PubMed]

49. Ruiz, E.; Ferro, V.R.; Palomar, J.; Ortega, J.; Rodríguez, J.J. Interactions of ionic liquids and acetone: Thermodynamic properties, quantum chemical calculations and NMR analysis. J. Phys. Chem. B 2013, 117, 7388-7398. [CrossRef] [PubMed]

50. Kurteva, V.; Atanassova, M.; Billard, I. NMR Study on the possible interactions between imidazolium based ionic liquids and extractants widely applied in solvent extraction and separation of f-ions. J. Solut. Chem. 2015, 44, 2416-2430. [CrossRef]

51. Cao, Y.; Xing, H.; Yang, Q.; Li, Z.; Chen, T.; Bao, Z.; Ren, Q. Biphasic systems that consist of hydrophilic ionic liquid, water, and ethyl acetate: The effects of interactions on the phase behavior. Ind. Eng. Chem. Res. 2014, 53, 10784-10790. [CrossRef]

52. Friesen, S.; Buchecker, T.; Cognigni, A.; Bica, K.; Buchner, R. Hydration and counterion binding of [C $\left.{ }_{12} \mathrm{MIM}\right]$ micelles. Langmuir 2017. [CrossRef] [PubMed]

53. Skarmoutsos, I.; Welton, T.; Hunt, P.A. The importance of timescale for hydrogen bonding in imidazolium chloride ionic liquids. Phys. Chem. Chem. Phys. 2014, 16, 3675-3685. [CrossRef] [PubMed]

54. Lee, B.-S.; Lin, S.-T. A priori prediction of the octanol-water partition coefficient (Kow) of ionic liquids. Fluid Phase Equilibr. 2014, 63, 233-238. [CrossRef]

55. Ropel, L.; Belvèze, L.S.; Aki, S.; Stadtherr, M.A.; Brennecke, J.F. Octanol-water partition coefficients of imidazolium-based ionic liquids. Green. Chem. 2005, 7, 83-90. [CrossRef]

(C) 2017 by the authors. Licensee MDPI, Basel, Switzerland. This article is an open access article distributed under the terms and conditions of the Creative Commons Attribution (CC BY) license (http:/ / creativecommons.org/licenses/by/4.0/). 